\title{
Modernisation de l'irrigation de surface : acquis et perspectives
}

\author{
Daniel Renault \\ ENGREF Centre Montpellier - BP 5093 - 34033 Montpellier Cedex 1 \\ Tél. : 67047100 - Fax : 67047101
}

\section{Introduction}

Environ 150000 ha irrigués du Sud-Est de la France utilisent des techniques dites de surface ou gravitaire; ces périmètres, pour la plupart très anciens sont implantés dans les vallées des fleuves méditerranéens. Si aujourd'hui, en France l'équipement de nouveaux périmètres utilisant ces techniques n'est pas à l'ordre du jour, le devenir des périmètres existants est posé. Il est posé en termes d'amélioration de la productivité agricole et en termes d'optimisation des ressources naturelles.

La modernisation des périmètres d'irrigation de surface peut être envisagée en faisant appel aux autres techniques d'irrigation, l'aspersion ou l'irrigation localisée, lorsque celles-ci sont économiquement et techniquement adaptées au contexte. Il est cependant des situations où l'irrigation gravitaire garde tout son intérêt (faible coût, insensibilité aux vents, ...) et pour lesquelles l'introduction de pratiques modernisées semblent être une réponse pertinente. C'est en s'appuyant sur de telles situations que la politique de modernisation de l'irrigation de surface s'est développée en
France au cours des années 80 en adaptant des techniques d'amélioration déjà éprouvées à l'étranger ou en développant ses propres technologies.

Des améliorations substantielles ont été enregistrées dans la pratique de l'irrigation de surface au niveau de la parcelle. Ces progrès visent à la fois à diminuer les charges de main-d'œuvre nécessaires à l'irrigation et à diminuer les pertes en eau.

La modernisation de l'irrigation de surface nécessite en outre de dépasser le cadre strict de la parcelle pour s'interroger sur ses implications collectives. En effet bon nombre de réseaux d'alimentation sont anciens, vétustes et nécessitent une adaptation pour les hisser au niveau des exigences d'une pratique moderne à la parcelle. Par ailleurs les ressources estivales en eau de certaines régions du sud de la France s'avèrent aujourd'hui fort dépendantes des aménagements et des pratiques liés à l'irrigation de surface. C'est donc à un niveau collectif que doivent être réfléchies et menées les actions complémentaires qui permettront véritablement à la modernisation de l'irrigation de surface d'exister en tant que schéma de développement cohérent.

\section{Surface irrigation modernization : acquirements and prospects}

Surface irrigation in France (15\% of the irrigated area had been recently modernized with particular emphasis on labor saving and water application efficiency increasing. $\star$ Improvements in field levelling and water distribution had occured. Many distributors have been successfully tested: gated pipe, layflat tubing, cable gation, low pressure buried pipe, ... However there still are improvments to make on the surface irrigation parameters assessment (inflow and irrigation time). $\star$ The rehabilitation of the distribution network to satisfy the modern surface irrigation requirements is often needed. $\star$ Last, surface irrigation modernization must also be debate in terms of water resources management at a regional scale. 

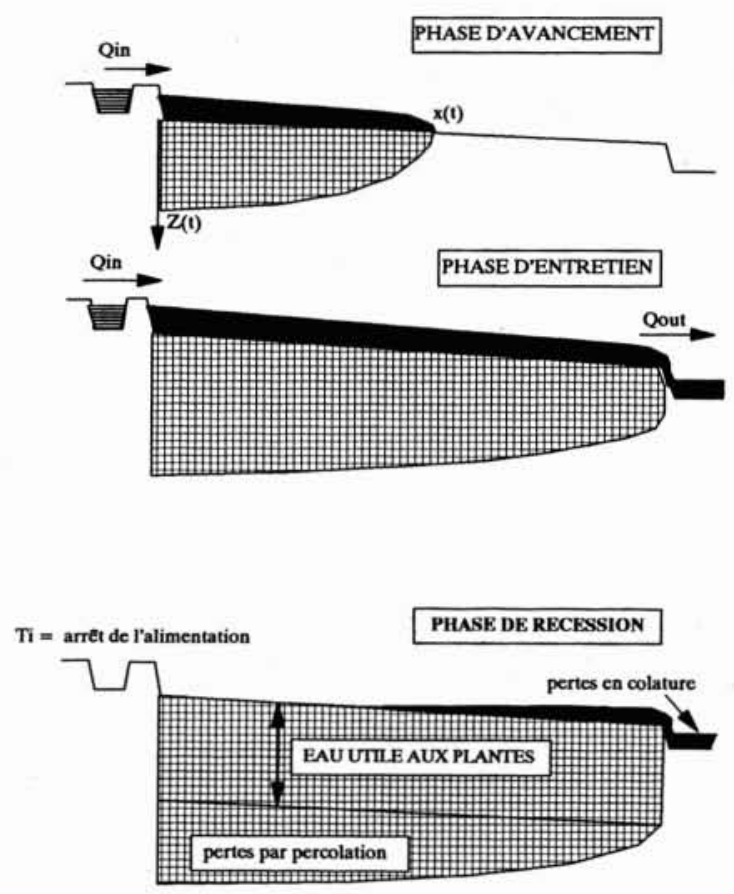

1. Schématisation des phases de l'irrigation de surface par ruissellement.

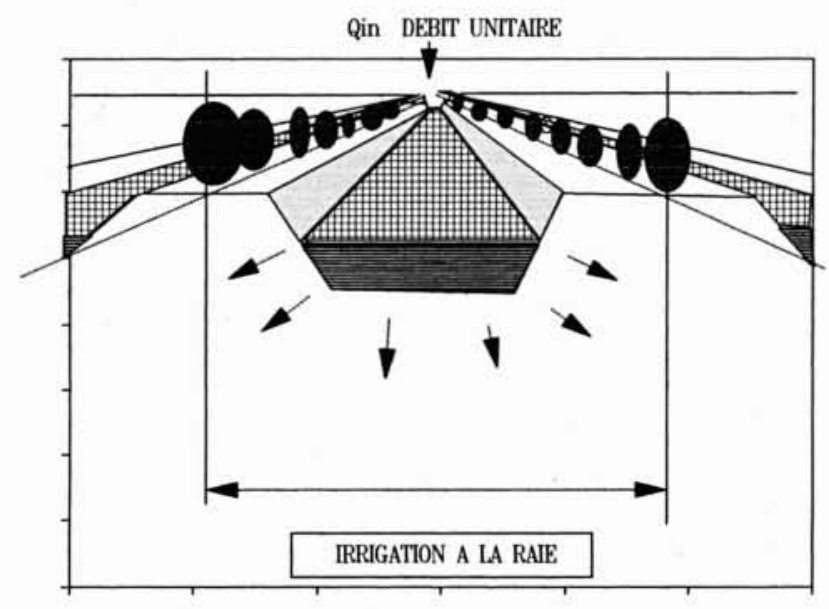

2. Schématisation de l'infiltration en irrigation à la raie.

L'une des spécificités de l'irrigation de surface par rapport aux autres modes d'irrigation (aspersion, irrigation localisée) est d'utiliser la surface du sol (d'où son appellation) comme lieu d'infiltration de l'eau d'irrigation et comme moyen de transport de celle-ci.

L'irrigation de surface comprend deux grands types de techniques :

1) La submersion concerne les techniques dites «de bassins ", généralement à fond plat. L'apport d'eau s'effectue par la mise en place rapide d'une lame d'eau importante et équivalente à la dose, sur la totalité de la surface du bassin, ou par maintien du niveau du plan d'eau dans le cas particulier de rizières. Dans cette technique, on considère que la différence de temps d'humidification susceptible d'être relevée entre différents points de la parcelle n'est pas importante.

2) Le ruissellement recouvre les techniques pour lesquelles l'apport d'eau se fait plus lentement. Le transport de l'eau au sein de la parcelle occasionne des différences de temps d'humidification importantes.

La plupart des techniques de ruissellement présentent les caractéristiques communes suivantes: un parcours en pente, une sortie aval pour évacuer le trop-plein de colature. Trois grands types peuvent être distingués : l'irrigation à la raie (le transport et l'infiltration s'effectuent dans un sillon aménagé entre les rangées de culture), plutôt adaptée aux grandes cultures, l'irrigation au calan (la bande de terre arrosée est comprise entre deux levées distantes de quelques dizaines de mètres) plutôt utilisée sur prairies ou luzerne; l'irrigation à la planche, version étroite du calan (la bande arrosée a quelques mètres de large) rencontrée en arboriculture.

Le mode de fonctionnement habituel de l'irrigation par ruissellement est le suivant (fig. 1):

- le module d'arrosage, débit disponible à la parcelle, est réparti à l'amont de la façon la plus uniforme possible, entre les raies, les calans ou les planches formant un même poste d'arrosage ; cette répartition uniforme doit également prévaloir au sein d'un calan ou d'une planche;

- la phase d'avancement de l'irrigation couvre la période durant laquelle le front avance jusqu'à l'aval de la parcelle ; - la phase d'entretien de l'irrigation dure entre le moment où le front atteint l'aval de la parcelle et celui où l'alimentation en eau sur le poste d'arrosage est coupée à l'extrémité amont de la parcelle ;

- enfin la phase de récession débute à l'arrêt de l'alimentation du poste et dure jusqu'à la disparition totale de l'eau en tout point du secteur de parcelle arrosé.

Dans ce mode de fonctionnement, le surplus de débit mis en jeu par rapport au cumul de l'infiltration sur le parcours se déverse en colature à l'aval du champ durant la phase d'entretien et celle de récession. On parle de pertes en colature.

Dans ce mode de fonctionnement également, le temps de présence de l'eau sur la partie amont du parcours est généralement plus élevé que sur la partie aval. La quantité 
d'eau infiltrée étant directement reliée au temps d'humidification, la satisfaction des quantités d'eau requises à l'aval est obtenue au prix de pertes en percolation (infiltration au-delà de la zone exploitée par les racines) sur le parcours amont (cf. fig. l).

La connaissance de l'infiltration est essentielle pour apprécier les quantités d'eau utiles aux plantes et dégager des consignes d'amélioration pour la conduite de l'irrigation. Or, l'infiltration est un processus physique complexe, excessivement variable dans l'espace et à diverses échelles de temps, et s'avère difficile à appréhender directement. En irrigation à la raie par exemple (fig. 2), l'infiltration est un processus bidimensionnel faisant intervenir les propriétés hydrodynamiques verticale et horizontale du sol en place.

\section{Paramètres d'arrosage (débit, temps d'irrigation) et efficience}

\subsection{Efficience de l'arrosage}

L'efficience d'un arrosage permet d'apprécier son niveau de performance. L'efficience appelée également rendement hydraulique représente le ratio entre la quantité d'eau utile aux plantes et celle mise en jeu.

Compte-tenu des pertes inévitables (colature, percolation), l'irrigation de surface offre un niveau de performance relativement bas (ordre de grandeur $50 \%$ ).

\subsection{Paramètres de choix d'un arrosage $\left(Q_{i n}, t_{i}\right)$}

\subsubsection{Le débit en tête, $Q_{\text {in }}$}

A un débit faible correspond une vitesse lente d'avancement du front occasionnant des pertes importantes par percolation à l'amont de la parcelle.

A l'opposé, un débit fort (dans la limite non érosive) permet au front d'atteindre très rapidement l'aval de la parcelle, ce qui a pour effet de diminuer les pertes par percolation mais par contre risque d'augmenter substantiellement celles par colature au cours de la phase d'entretien.

Le débit optimum doit ainsi être un compromis entre ces deux valeurs, cependant en raison du caractère éminemment variable de l'infiltration, le choix du débit reste encore bien souvent le résultat d'une démarche empirique.

Des méthodes d'optimisation des paramètres d'arrosage font actuellement l'objet de recherche-développement. Elles procèdent généralement à partir d'une évaluation de l'irrigation en temps réel (calage du modèle au cours de l'irrigation).

\subsubsection{Le temps d'irrigation}

Le temps d'irrigation peut être défini comme étant la durée de mise en eau de la raie ou du calan qui permet en tout point du parcours d'infiltrer la quantité d'eau requise, $\left(D_{n}\right)$ la dose nette.
Le temps d'irrigation dépend ainsi de trois termes :

- le temps d'humidification nécessaire à l'infiltration de la dose $t_{h}\left(D_{n}\right)$;

- le temps d'avancement $t_{a}(x)$, en un point d'abscisse $x$;

- le temps de récession $t_{r}(x)$, en un point d'abscisse $x$.

En irrigation par ruissellement, l'extrémité aval est généralement le secteur le moins arrosé. C'est donc en référence à ce point $(\mathrm{L})$ que l'on détermine le temps d'irrigation comme suit :

$$
T_{i}=t_{h}\left(D_{n}\right)+t_{a}(L)-t_{r}(L) .
$$

Pour des conditions aux limites identiques (débit $Q_{i n}$ ) ces trois termes sont susceptibles de varier de façon très conséquente au cours de la saison d'irrigation d'où une certaine difficulté dans la prédétermination du temps d'irrigation.

\section{Les améliorations au niveau de la parcelle}

De très nombreuses améliorations de la pratique traditionnelle de l'irrigation de surface ont été développées au plan mondial durant les dernières décennies. Elles correspondent à un enjeu essentiel pour l'alimentation de l'humanité, puisque la très grande majorité des superficies mondiales irriguées relèvent des techniques de surface. Ce constat concerne les pays en développement mais également certains pays développés. A titre d'exemple, citons le cas des USA où l'irrigation de surface couvre de 60 à $70 \%$ des 25 millions d'hectares irrigués.

En France, l'amélioration de l'irrigation de surface à la parcelle a fait l'objet d'études suivies depuis 1980. Ces techniques améliorées portent sur la gestion des débits, la mécanisation de la distribution, la maîtrise du nivellement et la conduite des irrigations.

\subsection{La gestion des débits}

\section{L'arrosage à débit constant en irrigation traditionnelle}

Le secteur arrosé (raie ou portion de calan) est alimenté durant la totalité de l'arrosage par une même valeur de débit, censée représenter un compromis visant à minimiser l'ensemble des pertes. Cette méthode est la plus simple mais n'est pas la plus efficace au plan des économies d'eau. Cependant bien calée, elle peut permettre des valeurs d'efficience dépassant légèrement les $60 \%$.

\section{L'arrosage à deux débits}

A un débit d'attaque élevé, permettant au front d'atteindre rapidement l'aval de la parcelle, succède un débit d'entretien beaucoup plus faible, limitant par là même les pertes en colature. La pratique du double débit peut améliorer notoirement les performances de l'irrigation.

\section{L'arrosage à débit varié décroissant}

Ce type d'arrosage résulte de l'utilisation d'un système 


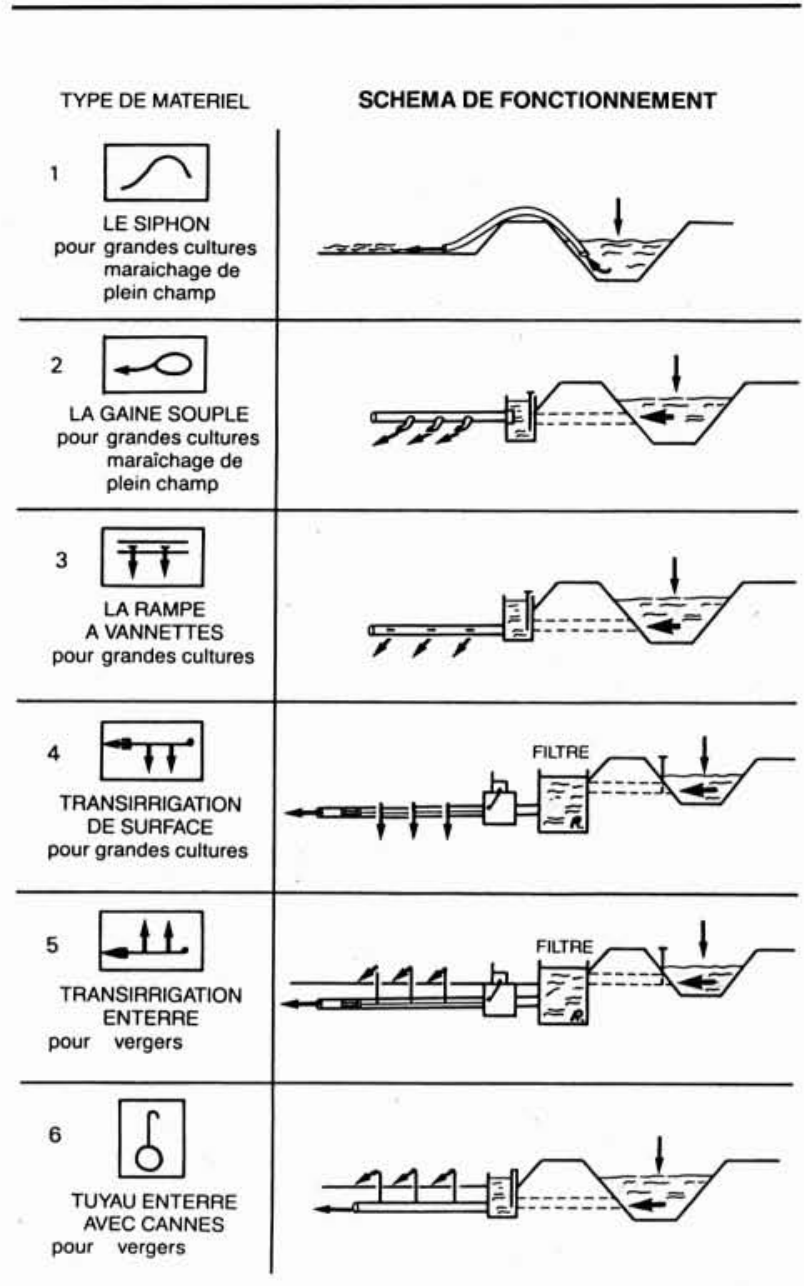

3. Les distributeurs de surface en irrigation à la raie.

Extrait de la plaquette « Modernisation de l'irrigation à la raie ", éditée par le Réseau national expérimentation démonstration secteur hydraulique agricole (RNEDHA) - B.P. 5095 - 34033 MONTPELLIER CEDEX 1 - Tél. : 67524343 Télex : $490990 \mathrm{~F}$.

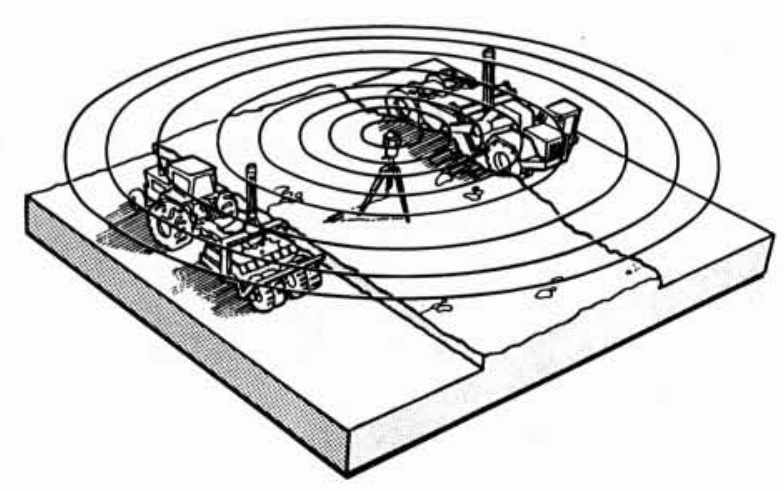

4. Nivellement par guidage laser. automatisé particulier: le transirrigation. A partir d'une valeur de débit d'attaque que l'on peut choisir au moment de la conception de l'appareil, le débit unitaire injecté dans la raie ou sur la portion de calan, diminue progressivement jusqu'à s'annuler. Il est permis d'atteindre avec ce procédé des efficiences supérieures ou égales à $80 \%$.

\section{L'arrosage par vagues}

Il consiste à faire alterner dans la même raie de courtes phases d'humidification avec de courtes phases de ressuyage. L'effet le plus spectaculaire est une augmentation notoire de la vitesse du front (en temps compensé) lors de l'avancement, ce qui a pour effet de réduire les pertes par percolation. En faisant varier la fréquence des cycles lors de la phase d'entretien, on induit également une diminution des pertes par colature.

\section{L'arrosage sur raies bouchées}

Dans ce mode d'apport les raies sont fermées à leur extrémité aval. Ce type d'arrosage est délicat à maîtriser en raison des risques d'ennoiement à l'extrémité aval, il est intéressant dans la mesure où il supprime les pertes en colature.

\subsection{La mécanisation de la distribution d'eau}

Dans l'irrigation traditionnelle de surface, la répartition du débit à l'amont de la parcelle est difficile à obtenir et relativement précaire en raison des phénomènes d'érosion intervenant aux droits des entrées d'eau sur un même poste (raie ou portion de calan). Ainsi, la présence permanente de l'irrigant est-elle quasiment nécessaire pour éviter toute dérive préjudiciable à l'uniformité d'arrosage.

L'utilisation de distributeurs mécanisés à l'amont de la parcelle est un élément capital pour garantir une répartition uniforme du débit et prévenir les dérives ultérieures. Ces distributeurs, réglés en début d'arrosage, peuvent être laissés sans surveillance. Ils économisent la main-d'œuvre, facilitent la conduite des arrosages et améliorent les performances de l'irrigation (fig. 3).

\section{Les siphons}

Le siphon est un tube de plastique coudé, de faible diamètre qui, une fois amorcé, assure le transfert du débit entre le portau (canal en terre implanté en partie amont de la parcelle) et la raie ou la portion de calan qu'il dessert. C'est un système mécanisé, peu onéreux, de mise en œuvre facile, qui nécessite une très faible charge en tête de parcelle.

\section{Les rampes à vanettes}

Le traditionnel portau à l'amont de la parcelle est remplacé par une canalisation munie de vannettes à ouverture réglable aux droits des raies à desservir. La rampe à vannette est d'une utilisation simple et d'une mise en fonctionnement très rapide. 


\section{Les gaines souples}

Ce système diffère peu du précédent: le transport et la distribution s'effectuent dans une conduite souple, très légèrement en charge, alimentant les raies au travers de manchettes à orifice réglable. Au repos, elle peut être franchie par les engins agricoles.

\section{Le transirrigation}

Une canalisation, positionnée avec une pente très précise en partic amont de la parcelle, assure le transport et la distribution de l'eau au travers d'orifices situés dans sa partic supérieure. C'est un distributeur entièrement automatisé.

La conduite est dimensionnée pour que la ligne d'eau de l'écoulement à surface libre se situe en régime normal sous le niveau des orifices. A l'intérieur de la conduite, un piston dont le mouvement est contrôlé à partir de l'ouvrage de tête, crée une zone de remous, met en charge un certain nombre d'orifices et alimente ainsi les raies ou les planches situées à son amont immédiat.

Le débit délivré sur chaque orifice décroit depuis une valeur maximale lorsque l'orifice est mis en eau jusqu'à une valeur nulle lorsque le train d'arrosage s'est déplacé vers l'aval. Cette modulation du débit améliore l'efficience de l'eau à la parcelle.

\section{Le tuvau enterré avec cannes}

Une conduite enterrée, implantée en partie amont de la parcelle, alimente des cannes de sorties. Généralement utilisées sur vergers, ces cannes de sortie sont implantées aux droits des rangées ; elles sont munies ou non de dispositif de réglage et de fermeture.

\subsection{La maîtrise du nivellement des parcelles}

Une topographie uniforme est indispensable à l'irrigation de surface à la fois pour générer un transport homogène au sein des secteurs irrigués, à la fois pour garantir des conditions d'infiltration identiques.

Le nivellement des parcelles bénéficie aujourd'hui des progrès liés à l'utilisation du laser rotatif pour guider les engins de terrassement. Un gain important de productivité et une meilleure précision dans le travail en ont résulté (fig. 4).

\subsection{La maîtrise de la conduite des irrigations}

Au même titre que les autres techniques, l'irrigation de surface bénéficie des progrès récents intervenus dans l'appréciation de la demande en eau des plantes et de l'état hydrique du sol. Ainsi l'usage des tensiomètres permet-il un contrôle a posteriori de la qualité de l'irrigation (diffusion latérale, répartition longitudinale, profondeur humidifiée). Il permet également de déclencher l'irrigation au moment opportun pour la culture.

\section{Les progrès restant à accomplir dans l'acquisition des données}

Les phénomènes physiques régissant l'irrigation de surface sont d'un abord généralement plus complexe que pour les autres techniques d'irrigation. Cela tient à la fois à la nature des phénomènes en jeu (transport à surface libre couplè au transfert d'eau en milieu non saturé) et à la grande variabilité de leurs caractéristiques physiques.

Ainsi la variabilité spatiale et temporelle de l'infiltration s'avère souvent trop importante et les facteurs qui la déterminent en trop grand nombre, pour que ce terme puisse faire l'objet d'une prédiction fiable à l'échelle de la parcelle.

L'amélioration des performances de l'irrigation de surface bute actuellement sur le problème de la mesure des éléments en jeu (débit, infiltration). Il n’existe pas de moyens simples, fiables et précis pour accéder à ces données. Or la connaissance de ces données est indispensable pour optimiser l'irrigation de surface et gérer au mieux les paramètres d'arrosages, le débit $Q_{i n}$ et le temps d'arrosage $T_{i}$, en fonction d'un objectif d'irrigation $\left(D_{n}\right)$.

Faute de pouvoir prédire correctement le fonctionnement de l'irrigation de surface, les recherches en cours se sont plutôt orientées vers une analyse en temps réel des phénomènes. Cela consiste notamment à interpréter le déplacement du front lors de la phase d'avancement pour en déduire certaines caractéristiques globales sur l'irrigation en cours, puis à modifier le réglage si besoin est, lors de la phase d'entretien et enfin prédire le temps $\left(T_{i}\right)$ nécessaire à l'infiltration de la dose $\left(D_{n}\right)$.

Le développement de ces méthodes d'optimisation (cf. Bibliographie) est en phase d'achèvement. Toutefois, leur application sur le terrain nécessite également de résoudre le problème de l'acquisition des données (débit, avancement du front). Ce problème devra être considéré comme prioritaire dans les recherches futures.

\section{Les enjeux de la modernisation de l'irrigation de surface au plan collectif}

Au plan national, le bilan de la décennic 80 en irrigation de surface peut être qualifié de positif. Sous l'impulsion du Ministère de l'Agriculture, de nombreux partenaires ont joint leurs forces pour mener à bien les expérimentations et validations nécessaires à l'introduction et au développement des nouvelles techniques évoquées précédemment.

Ces techniques modernes à la parcelle sont aujourd'hui maîtrisées, les matériels sont fabriqués industriellement et une politique de diffusion auprès des agriculteurs a été mise en place notamment au travers des actions menées par le RNEDHA (cf. légende de la fig. 4).

Cependant, si les besoins d'amélioration de cette technique traditionnelle sont bien réels, les opérations de modernisation et/ou réhabilitation des périmètres irrigués sont encore peu nombreuses et lentes à se mettre en œuvre. A l'heure où les économies d'eau et la protection de l'environnement sont des enjeux de plus en plus sensibles, il 
convient d'analyser les facteurs qui freinent l'évolution de l'irrigation de surface.

Après avoir maîtrisé les conditions de son succès au niveau de la parcelle et au niveau de l'exploitation, il apparaît aujourd'hui de plus en plus clairement que la modernisation de l'irrigation de surface se gagnera définitivement au plan collectif.

Ce constat peut être décliné à deux niveaux :

- collectif au sens du réseau d'alimentation du périmètre irrigué, et

- collectif au sens de la gestion des ressources et du milieu.

\subsection{Modernisation des réseaux de distribution}

La modernisation des infrastructures de distribution au sein des périmètres d'irrigation gravitaire est une double nécessité :

- d'abord parce qu'elle est susceptible de générer par elle-même d'importantes économies d'eau en supprimant les pertes d'eau, en ligne ou liées à la gestion physique du réseau. Il faut savoir que les infrastructures gravitaires sont pour la plupart anciennes et ne répondent plus nécessairement aux critères actuels d'efficacité soit par manque d'entretien soit tout simplement parce que les critères de conception ont changé ;

- ensuite pour améliorer la qualité du service fourni aux irrigants. En effet, l'expérience montre que la pratique moderne de l'irrigation de surface à la parcelle est étroitement tributaire d'une bonne qualité dans l'alimentation en eau de la parcelle.

L'usage des distributeurs de surface exige une eau :

- en légère charge $(0,1$ bar) pour faciliter l'équilibre hydraulique du distributeur ;

— propre, débarrassée des débris grossiers risquant d'obstruer les orifices ;

- livrée avec un débit le plus régulier possible afin de pouvoir laisser l'arrosage sans surveillance après le réglage initial (éviter les sous-irrigations ou les pertes trop abondantes).

Par ailleurs, un contrôle fin de l'irrigation, basé sur un suivi du bilan hydrique à la parcelle, requiert de pouvoir disposer de l'eau au moment opportun pour la culture. Cette exigence s'accommode mal du mode traditionnel de distribution de l'eau au sein des périmètres d'irrigation gravitaire à savoir « le tour d'eau ». Une évolution vers une plus grande souplesse dans l'accès à l'eau apparaît souhaitable.

Ainsi la pratique moderne de l'irrigation de surface à la parcelle ne saurait dans la plupart des cas s'envisager sans une modernisation conséquente du réseau d'alimentation.
Cela implique à la fois un coût d'investissement élevé et des délais importants dans la réalisation des opérations de modernisation.

Différentes approches de la modernisation des réseaux d'alimentation se sont faits jour ces dernières années. Parmi les solutions récemment développées, dans le Sud de la France, la technologie de transport Basse-Pression apparaît à bien des égards prometteuse. Cette technologie consiste à remplacer l'ancien réseau à surface libre par des canalisations enterrées fonctionnant à faible pression, soit à partir d'une station de pompage soit en tirant parti du dénivelé naturel du terrain. C'est dans ce dernier cas que la filière Basse-Pression semble être la plus avantageuse. Il est raisonnable de penser qu'à la fin de la décennie 90 un bilan tenant à la fois compte du coût de l'investissement et des frais de fonctionnement des réseaux modernes pourra être dressé à partir des expériences réalisées dans le Sud-Est de la France.

\subsection{Modernisation et gestion régionale des ressources en eau}

Pour une large part la modernisation de l'irrigation de surface répond à un souci d'économie d'eau à la parcelle, à savoir limiter les pertes en colature et par percolation. Cependant le cadre strict de la parcelle est quelquefois insuffisant pour analyser correctement la gestion de l'eau en irrigation de surface ; celle-ci doit également être envisagée dans un cadre régional au sens hydrologique du terme.

Etablis depuis des siècles pour certains, dans des régions à fort déficit hydrique estival, les périmètres d'irrigation de surface ont une influence non négligeable sur l'équilibre hydrogéologique de ces régions. Les pertes d'eau générées à la parcelle se retrouvent sur le bassin dans les réseaux hydrographiques de surface, ou dans les nappes souterraines. Des exemples récents ont montré comment l'abandon de l'irrigation de surface pouvait remettre en cause l'alimentation de certaines nappes alluviales dans le Sud-Est de la France et pénaliser soit d'autres irrigants, soit d'autres usages de l'eau. Inversement, il est certain également que les percolations en provenance des terres agricoles peuvent représenter une source de dégradation de la qualité des ressources souterraines. En pratique il n'est guère possible de définir une règle générale en ce qui concerne l'impact et le devenir des "pertes d'eau " à la parcelle. En conséquence, l'approche régionale de la gestion de l'eau dans la modernisation de l'irrigation de surface doit prendre en compte les spécificités locales du milieu et des pratiques agricoles afin de régler au mieux la satisfaction des besoins régionaux (production agricole, alimentation en eau, protection du milieu). 


\section{Bibliographie sommaire}

Renault D. (1987). — " Automatisation de l'irrigation de surface : le bain de jouvence." Article publié dans la revue Génie Rural nº 4, avril 1987 (4 pages).

« Les nouveautés dans l'irrigation à la parcelle » (collectif). Article publié dans la revue Génie Rural, juillet 1987.

GoÉnaga M., Renault D. (1987). - « Réhabilitation des périmètres irrigués de Tarascon. Les Réseaux collectifs BassePression au service d'une irrigation gravitaire performante et économe. "ICIID XIII Congrès, Rabat 87, Q.40 - R 57, pp. 868-878.

Renault D. (1988). - " Modernization of furrow irrigation in the South-East of France : automation at field level and its implications." Report in "Irrigation and Drainage Systems ", n² 2, 1988, pp. 229-240.

Baudequin D. (CEMAGREF), Galand A. (SCP), Renault D. (ENGREF) (1988). - "Les Réseaux Basse-Pression en irrigation de surface », 100 pages. Compte rendu de l'atelier Réseaux Basse-Pression organisé à l'ENGREF en novembre 1988.

Mailhol J.C., Bertome P., Renault D. (1989). - « Les Paramètres de choix en irrigation de surface. " Invention au Congrès International U.N. - CEE, Avignon France, septembre 1989.

Renault D. (1980). - " Low pressure techniques in surface irrigation rehabilitation in South-Eastern France " (11 pages). Proceedings of the International Conference on Water and Waste Water. Barcelona, April 1990.

ReNAULT D. (1991). - Modélisation hydrologique en irrigation de surface: Analyse de la linéarisation de la vitesse du front d'avancement : ALIVE. Thèse de $3^{e}$ cycle USTL Montpellier ENGREF, 200 pages.

Renault D., Wallender W. (1992). - «Alive» Advance Linear Velocity: a flow rate balance theory for surface irrigation. Journal of Irrigation and Drainage Engineering, vol. $118, \mathrm{n}^{\circ} 1$, January/February 92.

\section{Documentaires vidéo}

[1] " La modernisation de l'irrigation de surface dans le Sud-Est de la France," Vidéo VHS Secam $17 \mathrm{~min}$. Production ENGREF/CEMAGREF.

[2] "Techniques Modernes d'irrigation de Surface. " Vidéo S. VHS 20 min. Production ENGREF/RNEDHA. 


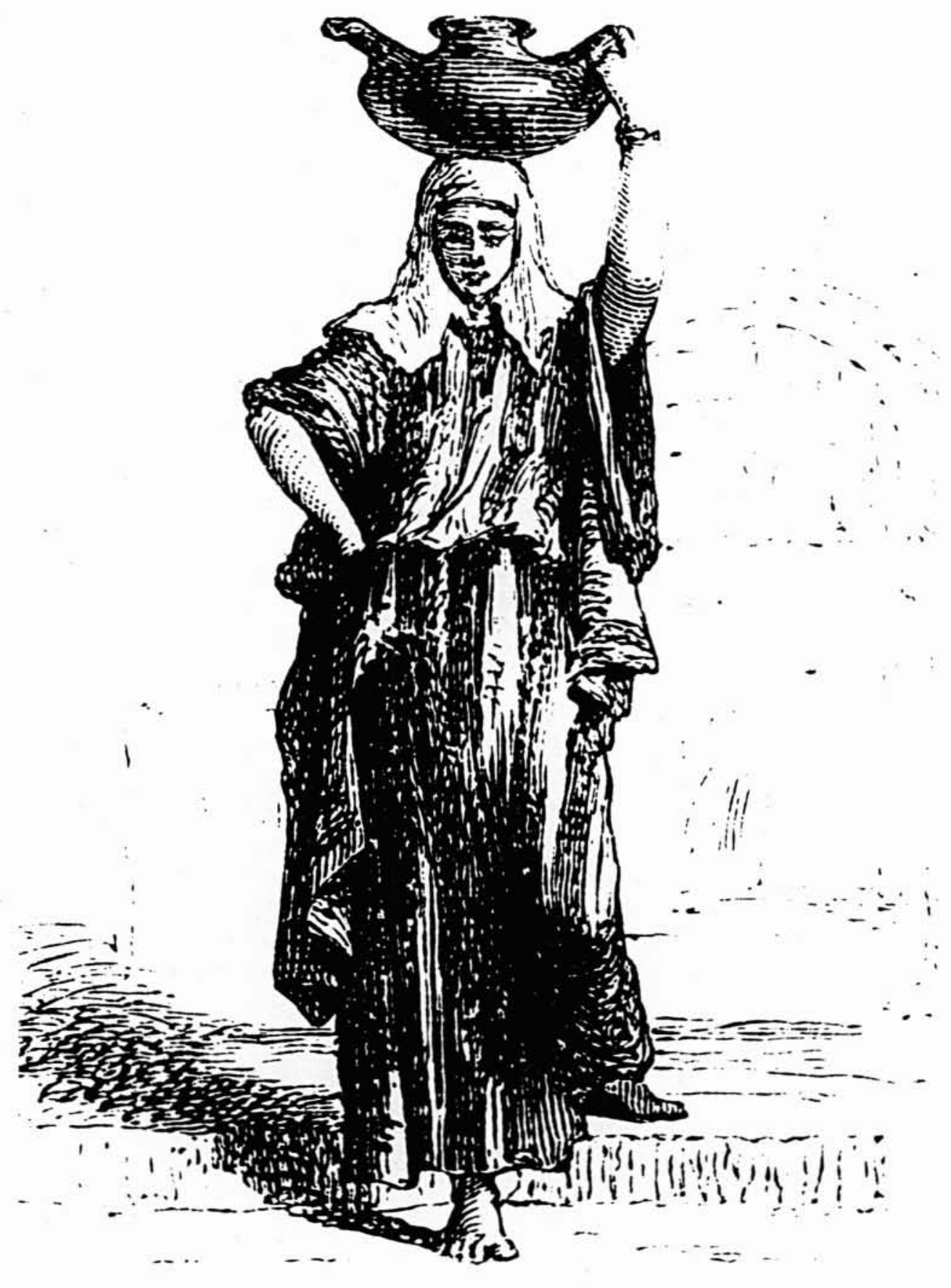

